\title{
Precision short-pulse
} damage test station utilizing optical parametric chirpedpulse amplification

I. Jovanovic, C. Brown, B. Wattellier, N. Nielsen, W. Molander, B. Stuart, D. Penningtor and C. P. J. Barty

For submission to Review of Scientific Instruments

April 7, 2004 
This document was prepared as an account of work sponsored by an agency of the United States Government. Neither the United States Government nor the University of California nor any of their employees, makes any warranty, express or implied, or assumes any legal liability or responsibility for the accuracy, completeness, or usefulness of any information, apparatus, product, or process disclosed, or represents that its use would not infringe privately owned rights. Reference herein to any specific commercial product, process, or service by trade name, trademark, manufacturer, or otherwise, does not necessarily constitute or imply its endorsement, recommendation, or favoring by the United States Government or the University of California. The views and opinions of authors expressed herein do not necessarily state or reflect those of the United States Government or the University of California, and shall not be used for advertising or product endorsement purposes. 


\title{
Precision short-pulse damage test station utilizing optical parametric chirped-pulse amplification
}

\author{
Igor Jovanovic, Curtis Brown, Benoit Wattellier, Norman Nielsen, \\ William Molander, Brent Stuart, Deanna Pennington, and C. P. J. Barty \\ National Ignition Facility Programs, Lawrence Livermore National Laboratory, \\ Mail Code L-490, 7000 East Avenue, Livermore, California 94550 \\ jovanovic1@llnl.gov
}

The next generation of high-energy petawatt (HEPW)-class lasers will utilize multilayer dielectric diffraction gratings for pulse compression, due to their high efficiency and high damage threshold for picosecond pulses. The peak power of HEPW lasers will be determined by the aperture and damage threshold of the final dielectric grating in the pulse compressor and final focusing optics. We have developed a short-pulse damage test station for accurate determination of the damage threshold of the optics used on future HEPW lasers. Our damage test station is based on a highly stable, high-beam-quality optical parametric chirped-pulse amplifier (OPCPA) operating at $1053 \mathrm{~nm}$ at a repetition rate of $10 \mathrm{~Hz}$. We present the design of our OPCPA system pumped by a commercial Qswitched pump laser and the results of the full system characterization. Initial short-pulse damage experiments in the far field using our system have been performed. 


\section{INTRODUCTION}

A strong motivation for development of the next generation of high-energy petawatt (HEPW) lasers exists. ${ }^{1}$ Important applications enabled by HEPW lasers include advanced radiography with high-energy x-ray sources, ${ }^{2}$ studies of hot dense matter, ${ }^{3}$ and demonstration of fast ignition. ${ }^{4}$ The future HEPW-class lasers will be based on chirpedpulse amplification in Nd-doped phosphate glass at a center wavelength of $1053 \mathrm{~nm}$ and will produce >1-ps, multi-kJ pulses. The high-energy requirement for HEPW pulses represents an improvement of at least one order of magnitude over the state-of-the-art lasers in this pulse width regime. Production of HEPW pulses remains a technical challenge, the primary concern being the high fluence and short pulse width experienced by the final HEPW optics, i. e. the final compressor grating and beam transport and focusing optics. Those components are currently being designed and optimized at several institutions. ${ }^{5}$ This development process requires a precise instrument to produce consistent pulse fluences and focused intensities for determination of damage mechanisms and optical damage thresholds.

Past experimental studies of laser damage in metals and dielectrics using long

(nanosecond) pulses have been detailed and numerous. ${ }^{6-11}$ In the long-pulse regime, laser damage threshold is associated with thermal deposition and conduction, leading to irreversible modification of the material such as melting or fracture through induced stress. Systematic studies of interaction of short $(<20 \mathrm{ps})$ laser pulses with dielectric materials have been more recent ${ }^{12-15}$ and concurrent with the development of high-peak power short-pulse laser technology. While the damage mechanisms peculiar to long laser 
pulses are considered to be well understood, some mechanisms that govern interactions of short pulses with dielectrics have been elucidated only recently and are believed to be fundamentally different. It is thus of great importance to perform additional controlled studies at short pulse duration. These studies will be instrumental to improvement of high-power, short-pulse laser technology.

Experimental studies of short-pulse damage have been hampered by the lack of a stable, reliable, convenient, and well-characterized laser source capable of producing pulses with sufficient fluence and adequate pulse duration at moderate repetition rates. This is particularly the case with damage studies performed at the center wavelength near $1.05 \mu \mathrm{m}$, characteristic of amplification in Nd:glass lasers. With proliferation of petawattclass lasers based on chirped-pulse amplification in Nd:glass, damage studies of their optical components such as diffraction gratings at the design center wavelength will be required.

The requirements for a damage test laser source and setup are determined from the desired experimental conditions. It is currently believed that the mechanisms governing short-pulse damage are more deterministic than the mechanisms governing long-pulse damage. The accuracy of the short-pulse damage measurement is therefore directly related to shot-to-shot variations of experimental parameters, such as energy, pulse width, and focal spot size and shape. Here we summarize the resulting requirements imposed on the damage test setup:

1. Diffraction gratings and optical coatings used for HEPW are designed for a center wavelength of $1053 \mathrm{~nm}$, and their performance is highly dependent on the center 
wavelength. As a result, it is required that the damage test source operates at the design center wavelength of $1053 \mathrm{~nm}$.

2. Previous studies of short-pulse surface damage of polished fused silica indicate that damage thresholds near $5 \mathrm{~J} / \mathrm{cm}^{2}$ are expected for 10 -ps pulses centered near $1.05 \mu \mathrm{m} .{ }^{16}$ Since it is expected that the damage thresholds of dielectric diffraction gratings written on fused silica substrates are somewhat lower than the damage threshold of fused silica due to field enhancement effects, it is required that the fluence attainable using the damage test laser source be near $5 \mathrm{~J} / \mathrm{cm}^{2}$.

3. Diffraction gratings under study have groove spacings on the order of $1 \mu \mathrm{m}$. For a well-characterized damage test, illumination of hundreds of grooves is necessary. This, in combination with the minimum fluence specification, leads to the requirement for multi-millijoule pulse energies.

4. In order to obtain reliable statistical data on laser damage and possible laser conditioning effects, moderate $(>1 \mathrm{~Hz})$ repetition rate is desired.

5. High pulse-to-pulse energy stability is required to obtain accurate damage threshold data on a multi-shot basis.

6. High pulse width stability is required, leading to the requirement for stable spectral amplitude and phase.

7. High beam quality is desired in order to minimize hot spot peak fluence variations on a shot-to-shot basis.

8. High-accuracy, high-resolution measurements must be performed to determine pulse characteristics in space and in time. 
There are several possibilities for the damage test laser source. The obvious choice is a sub-scale Nd:glass system; however, Nd:glass is a poor thermal conductor and is therefore constrained to low repetition rate operation. Operation at low repetition rate is inconvenient and cannot produce reliable optic lifetime measurements. Off-centerwavelength amplification in Ti:sapphire has been previously used for short-pulse damage studies at $1.05 \mu \mathrm{m},{ }^{16}$ but it suffers from complicated architecture, requires a large amplification length due to low gain, and produces relatively high shot-to-shot energy variations.

Optical parametric chirped-pulse amplification (OPCPA) ${ }^{17}$ has been previously proposed $^{18}$ and demonstrated ${ }^{19}$ as a route to high peak power pulse production because of its potential high energy scalability and broad amplification bandwidth in type I phasematched nonlinear crystals such as beta-barium borate (BBO), lithium borate (LBO), and potassium dihydrogen phosphate (KDP). Favorable properties of nearly degenerate OPCPA can be also exploited at lower energy. Here, OPCPA can be used as a front end for Nd:glass high-peak-power, short-pulse lasers using simple commercial pump lasers $^{20,21,22}$, or using pump lasers specially optimized for OPCPA by spatiotemporal shaping. ${ }^{23,24}$ For low-energy, <millijoule-scale, high repetition rate systems, OPCPA in quasi-phase-matched materials has been developed in both nondegenerate ${ }^{25}$ and degenerate ${ }^{26,27}$ configurations.

Multi-millijoule OPCPA development up to date has been primarily driven by the replacement of Ti:sapphire front end technology at $1053 \mathrm{~nm}$ for front ends of high energy Nd:glass lasers. When used as a front end, OPCPA technology has multiple advantages over regenerative amplifiers based on Ti:sapphire. The advantages include simplicity, 
broad spectral bandwidth, high gain, high prepulse contrast, negligible thermal load, and elimination of electro-optic modulators. Our selection of OPCPA over Ti:sapphire and Nd:glass technology for short-pulse damage testing has been motivated by these advantages, allowing development of a relatively simple and reliable short-pulse damage test laser suitable for repetitive pulse damage studies.

A significant interest also exists in the laser community with respect to the use of OPCPA as reliable experimental tool. In this paper we report, to our knowledge, the first use of an OPCPA petawatt-type front end system for short-pulse damage studies. The design of a highly stable, nearly degenerate, OPCPA based on a commercial pump laser is presented, followed by characterization of several aspects of its operation directly relevant for damage testing experiments. Description of the precision, short-pulse damage test station built around the OPCPA system is given. Finally, examples of initial damage studies using OPCPA-based short-pulse damage test station are shown. Alternate uses of OPCPA technology include broad-bandwidth, high-contrast front ends for Petawatt-class Nd:glass lasers, ${ }^{24}$ generation of high peak power pulses through energy scaling, ${ }^{18}$ and high average power short-pulse generation using high repetition rate pump lasers. ${ }^{25}$

\section{OPCPA SYSTEM DESIGN AND OPERATION}

Our OPCPA system (Fig. 1) starts with a commercial Nd:glass oscillator (TimeBandwidth Product GLX-200), which delivers 250-fs pulses centered at $1053 \mathrm{~nm}$ at a repetition rate of $\sim 100 \mathrm{MHz}$. The spectral bandwidth of the oscillator is 7-nm FWHM. Pulses pass through a Faraday isolator and a collimating 4-m focal length lens, and are 
subsequently injected into a pulse stretcher. ${ }^{28}$ The stretcher utilizes a 20-cm width diffraction grating with a groove density of $1480 \mathrm{~mm}^{-1}$. A plano-convex lens is used to provide image flipping in the stretcher, which leads to spectral and spherical aberrations. The pulse stretcher clips the spectral content of the oscillator with a full-width of $16 \mathrm{~nm}$. The measured efficiency of the stretcher is $42 \%$, which results in $1-n J$ output pulses. The measured output pulse width of the stretcher is $1.3 \mathrm{~ns}$ FWHM, with a total grating separation in the stretcher of $7.6 \mathrm{~m}$ and an incident angle of $47.5^{\circ}$.

The output 4-mm FWHM diameter beam from the stretcher is introduced into a pulse selector, consisting of a Pockels cell and a pair of polarizers. While the external pulse selection is not essential in OPCPA due to the pulse-selective property of the pump pulse, the use of Pockels cell is desirable in our system, since it reduces the background level at the experimental focal spot. The fall and rise time of the Pockels cell driver limits the pulse slicing to a minimum of 5 pulses, which are injected into the OPCPA system. The timing system utilizes a master clock signal at $10 \mathrm{~Hz}$, provided by a Stanford Research Systems DG-535 signal generator. The master clock signal is used in coincidence with the diode signal from the oscillator to generate a trigger signal for the pump laser and diagnostics. The measured jitter of our electronic triggering system is $<200$ ps rms.

Our pump laser is a commercial, Q-switched, Nd:YAG laser (Continuum Powerlite Plus), providing 1.5-J, 532-nm pulses at a repetition rate of $10 \mathrm{~Hz}$. The laser is injection seeded, resulting in single longitudinal mode operation. The motivation for pumping OPCPA with single longitudinal mode pulses is twofold. Firstly, OPCPA as an instantaneous frequency conversion process is highly sensitive to temporal modulations 
of the pump pulse. Since the stretched signal pulse is chirped, pump temporal modulation is imprinted onto the amplified pulse spectrum. Secondly, OPCPA systems often run at high fluences, which can be relatively close to the damage threshold of nonlinear crystals and other optics. Smooth pump temporal profile, such as the one obtained from a laser operating with single longitudinal mode, reduces the probability of optical damage to nonlinear crystals. Operation close to the damage threshold is desirable, since it reduces the necessary gain length for constant gain, while it increases spectral, angular, and temperature bandwidth of the amplifier by reducing the accumulated phase mismatch.

Our pump laser produces 5.7-ns FWHM pump pulses from a Gaussian optic unstable resonator, resulting in a bullet-like spatio-temporal pulse shape ${ }^{21}$. The calculation of extractable pump energy has been performed by integrating the measured spatiotemporal evolution of the pump laser in a 3-ns window centered at the pump pulse temporal peak, and defined by the stretcher spectral bandpass and dispersion. It has been calculated that $18 \%$ of the total pump pulse energy is available in this window. The integrated transverse shape of the pump pulse is a supergaussian (Fig. 2), A typical timing jitter of our pump laser and electronics system is $\pm 1 \mathrm{~ns}$ rms.

The pump laser output is split into two beams: a $70-\mathrm{mJ}$ beam is used to pump the preamplifier, while a $700-\mathrm{mJ}$ beam is used to pump the power amplifier, with the remaining pump energy not used in the OPCPA system. The energy of both pump paths can be continuously adjusted using a waveplate and a polarizer, which allows gradual increase of pump power and reduced probability of optical damage. To increase our system reliability and reduce damage susceptibility, we have additionally derated previously measured damage thresholds of our nonlinear crystal coatings and optics by a 
factor of $>3$, compensating for the reduced nonlinear drive with longer nonlinear crystal lengths. While this leads to reduction of the spectral bandwidth, the intrinsic spectral bandwidth of the preamplifier and the power amplifier $(>100 \mathrm{~nm} \cdot \mathrm{cm})$ is still much greater than the input spectral bandwidth.

The diameter of the $70-\mathrm{mJ}$ beam is reduced to $\sim 2.2 \mathrm{~mm}$ using a 5:1 telescope, and the beam profile present on the frequency-doubling crystal in the pump laser is relay imaged to the center of the preamplifier length. The preamplifier consists of three $15-\mathrm{mm}$ long $\mathrm{BBO}$ crystals. Each crystal has a $2^{\circ}$-wedged back surface and is antireflection coated for pump and signal wavelengths, which increases efficiency and reduces parasitic reflections which would otherwise be amplified. We have selected BBO as our amplifying medium for its favorable thermomechanical properties and high effective nonlinearity of $2 \mathrm{pm} / \mathrm{V}$. The two major disadvantages of $\mathrm{BBO}$ are the large birefringent walk-off angle of $3.2^{\circ}$ and high angular sensitivity of $0.4 \mathrm{mrad} \cdot \mathrm{cm}$. Birefingent walk-off reduces the maximum conversion efficiency, particularly when small-diameter beams are used. However, our conversion efficiency is primarily determined by the amplification in the power amplifier. As a result, birefringent walk-off in the preamplifier has no significant impact on the overall conversion efficiency of the system. The benefit of the large effective nonlinearity of $\mathrm{BBO}$ is immediately obvious: with the chosen low pump intensities of $<200 \mathrm{MW} / \mathrm{cm}^{2}$, the required gain length of a noncritically-phase-matched crystal such as LBO becomes prohibitively long due to much lower nonlinearity. We use three BBO crystals in the preamplifier in a walk-off compensating scheme ${ }^{29}$ by arranging the consecutive crystal Y-axes in antiparallel geometry. As a result, the overlap of signal, idler, and pump is improved, and the total birefringent walk-off of the entire crystal 
assembly is equal to the walk-off of one crystal. The external noncollinear angle between the signal and idler is $0.5^{\circ}$ in the crystal principal plane, which adds additional $0.2 \mathrm{~mm}$ of walk-off over the length of the preamplifier gain length.

If the measured spatiotemporal shape of the pump pulse is taken into account, the calculated pump peak intensity in the preamplifier is $180 \mathrm{MW} / \mathrm{cm}^{2}$. We have obtained output power of $\sim 3 \mathrm{~mJ}$ from the preamplifier. Due to the relative proximity of the three $\mathrm{BBO}$ crystals and the lack of spatial filtering, parametric fluorescence is the limiting factor in producing more gain from the preamplifier. With the chosen pump intensity, our total measured fluorescence from the preamplifier is $<1 \%$ of the total measured pulse energy.

The output of the preamplifier is resized by a 1:10 beam expanding telescope to $\sim 15$-mm diameter, and a hard $250-\mu \mathrm{m}$ diameter aperture at the focal spot in the telescope is used to separate the amplified signal from the idler and from the majority of parametric fluorescence. The resulting signal pulse is injected as seed into the power amplifier.

The power amplifier consists of two $10 \times 10 \times 12-\mathrm{mm}^{3} \mathrm{BBO}$ crystals arranged in a walk-off compensating scheme. The peak pump intensity in the power amplifier is 170 $\mathrm{MW} / \mathrm{cm}^{2}$, resulting in 55-mJ amplified signal pulses with noncollinear angle of $\sim 1^{\circ}$. The resulting amplified pulse spectrum is shown is Fig. 3 at two different amplification levels, along with the input oscillator spectrum. Spectral broadening is the result of pump depletion at the temporal part of pump pulse which coincides with the peak of the signal spectrum. Since the pump pulse envelope varies slowly over the duration of the stretched signal pulse, it can be regarded as nearly constant. This results in earlier pump depletion occurring at the peak of the signal spectrum. Slower conversion and the onset of signal- 
to-pump backconversion in the pulse center are coincident with nearly undepleted gain in the pulse spectral wings, resulting in spectral broadening at FWHM. The broadened spectrum of the signal pulse contains sharp clip-off points at the point of stretcher bandpass. When driven deep into backconversion by increasing the pump intensity in the power amplifier, the amplified spectrum has a pronounced dip in the center, where backconversion by sum-frequency generation of signal and idler is strongest. The maximum conversion efficiency in the power amplifier is $\sim 16 \%$ to signal and idler, or $\sim 87 \%$ of the calculated available pump energy in the 3-ns temporal window.

Our amplified pulses are expanded using a 1:2 beam telescope to alleviate possible compressor grating damage. After pulse compression, it is desirable to obtain information on the pulse width and pulse shape for the entire range of pulse widths used for damage testing ( 0.5-10 ps). While simple intensity autocorrelation is adequate for providing pulse width information for pulses shorter than $\sim 1 \mathrm{ps}$, it becomes more problematic in the range 1-10 ps due to the large spatial window which exceeds the autocorrelator crystal aperture. In addition, we desire high dynamic range information about the pulse wings. Scanning cross-correlation is more suitable for measurements of longer pulse widths, but it requires a short probe pulse in addition to the 1-10 ps pulse that under characterization. Our compression scheme is designed to result in two pulses: a short, near-trasformlimited probe pulse, and a longer, energetic pulse used for damage testing. This is accomplished by introducing the expanded beam into the double pulse compressor (Fig. 4). The double pulse compressor consists of a $1480 \mathrm{~mm}^{-1}$ diffraction grating at an angle of $47.5^{\circ}$, vertical roof mirror, and two sets of vertically offset, horizontal, $90^{\circ}$ folding mirrors. Each of the two sets of horizontal fold mirrors is mounted on a separate 
translation stage, allowing independent adjustment of effective grating separation for two beams.

The pulse input into the double compressor is split using a 5\% beamsplitter, and the resulting two pulses are injected into the compressor with a vertical-parallel separation of $5 \mathrm{~cm}$. The high-energy compressed pulse is adjusted to the desired pulse width for experiments, while the low-energy pulse is compressed to its transform limit and used for temporal diagnostics of the high-energy pulse when its pulse width is greater than $\sim 1$ ps. The low-energy pulse acts like a fast probe and samples the high-energy pulse in a scanning, second-order cross-correlator. The cross-correlator utilizes a calibrated motorized translation stage for adjustment of the short-pulse diagnostic delay with respect to the high-energy long pulse. Two beams are crossed at a $10^{\circ}$ angle in a thin BBO crystal. The resulting cross-correlation signal and the two frequency-doubled pulses corresponding to two fundamental pulses are collected by three fast $(<200 \mathrm{ps})$ diode detectors. The maximum of the cross-correlation signal is normalized to the geometric mean of the maxima of two second-harmonic signals, reducing the noise that originates from the energy instability and possible fluctuations of the pulse width of the short diagnostic pulse. Characterization of these fluctuations has been performed and is described in Section 4.

\section{SHORT-PULSE DAMAGE TEST SETUP}

\subsection{Description of damage test setup}

A schematic of our short-pulse damage test setup is shown in Fig. 5. Diffraction gratings and optics damage thresholds and efficiencies can be strongly polarization- 
dependant. It is therefore important to have a clearly defined and highly polarized pulses incident onto the damage test sample. The horizontally polarized pulse emerging from the pulse compressor is incident onto a half-waveplate and a polarizer pair, which produces vertical linear polarization. The measured polarization purity is better than 99\%. A 5\% beamsplitter is used to direct a small amount of the energy into a pyroelectric energy meter $\mathrm{EM}_{1}$ through a focusing lens $\mathrm{L}_{1}$. The energy meter $\mathrm{EM}_{1}$ is calibrated to measure the energy incident on the sample by inserting a second, absolutely calibrated, pyroelectric meter in front of the sample. The beam transmitted through the beamsplitter is directed though a 2-m focal length lens $L_{2}$ onto the damage test sample. A focal length of $2 \mathrm{~m}$ was selected to avoid the effects of self-focusing of short pulses in air, which are proportional to the Rayleigh range of the focused beam. This selection of the f-number of approximately f/100 leads to focal spots of $\sim 250 \mu \mathrm{m}$ diameter on the sample. For longer focal lengths and large focal spots, it is necessary to use vacuum focusing to avoid accumulation of nonlinear effects (this is discussed in the following section).

The test sample is positioned at the beam focus and mounted on a rotation stage, which allows adjustment of the angle of incidence with an accuracy of $<0.5^{\circ}$. Transverse and longitudinal movement of the sample is also possible using precision micrometer stages. The beam diffracted from the sample is imaged using a $50-\mathrm{cm}$ lens $\mathrm{L}_{3}$ onto a 16bit CCD camera for measurement of the focal spot beam profile. A fraction of the diffracted beam is split using a beamsplitter and directed into a pyroelectric energy meter $\mathrm{EM}_{2}$. The drop in the ratio of the diffracted to incident energy is used as one of the indicators of a damage event. This can be correlated also to the observation of damage features on the beam recorded on the 16-bit CCD camera. Diffraction and reflection 
efficiency and absorption of the samples can be determined by measurement of the energy balance using absolutely calibrated energy meters $\mathrm{EM}_{3}$ and $\mathrm{EM}_{4}$ for reflected and transmitted pulses, respectively. Additional studies of the damage morphology are performed using a Nomarsky microscope.

\subsection{Estimate of self-focusing effects in the damage test setup}

An important consideration for short-pulse damage testing which is absent in longpulse damage testing is the transverse nonlinear phase accumulation and subsequent selffocusing. Nonlinear phase accumulation or B-integral is the result of the nonlinear index of refraction of material that the intense laser beam traverses, including bulk optics and air. It was found numerically and experimentally in our setup that self-focusing represents an important design consideration. If self-focusing is present and not accounted for by measuring the spot size and beam spatial profile as a function of energy, damage thresholds measurements can yield erroneously low values.

When a beam propagates through a nonlinear medium with nonlinear refractive index $n_{2}$, it accumulates nonlinear phase along infinitesimally short distance $d z$ :

$$
d \varphi_{N L}=\frac{2 \pi}{\lambda} n_{2} \frac{F(z)}{\tau} d z
$$

where $\varphi_{N L}$ is the nonlinear phase, $\lambda$ is the wavelength, $F$ is the beam fluence, and $\tau$ is the pulse width. When the focusing effect is stronger than diffraction, whole-beam selffocusing occurs. Well above this threshold, small-scale self-focusing amplifies ripples in the intensity profile and the beam breaks up into small filaments. 
In our system, B-integral is dominated by the contribution of self-focusing in the air during slow focusing of the beam from the final lens $\mathrm{L}_{2}$ (Fig. 5) to the damage test sample. For a Gaussian beam, the accumulated nonlinear phase, i. e. B-integral $\left(\Phi_{N L}\right)$ is

$$
\Phi_{N L}=\int_{-\infty}^{0} d \varphi_{N L}=\frac{\pi^{2}}{\lambda} n_{2} \frac{F_{\max }}{\tau} z_{R}
$$

where $F_{\max }$ is the peak fluence without self-focusing, and $z_{R}=\pi w_{0}{ }^{2} / \lambda$ is the Rayleigh range of the beam with spot size $w_{0}$. For a typical upper-bound fluence damage threshold on the order of $5 \mathrm{~J} / \mathrm{cm}^{2}$ and 10-ps pulses, this corresponds to $\Phi_{N L} \sim 0.1$ for a selection of spot size $w_{0}=125 \mu \mathrm{m}$. We can define this as an upper bound of the Rayleigh range, or the maximum spot size allowed in our system for which B-integral effects are negligible. As a consequence, short-pulse damage tests conducted in air in our setup are limited to spot sizes of $\sim 125 \mu \mathrm{m}$. In order to perform short-pulse damage tests over greater sample area and using higher pulse energies, it is mandatory to propagate the laser beam in vacuum to avoid nonlinear effects (see Fig. 6).

\section{OPCPA SYSTEM PERFORMANCE CHARACTERISTICS}

\subsection{Energy stability}

High pulse-to-pulse stability is essential for high-accuracy multi-shot damage threshold measurements. We have performed pulse-to-pulse energy stability measurements by recording individual shot energies for 600 shots (1 minute of continuous operation). The results are shown in Fig. 7 and indicate that our pulse-to-pulse stability was $\pm 1.9 \%$ rms. We measured the peak power stability of the pump laser using a 200-ps diode. Peak power stability of the pump pulse is more relevant for understanding the OPCPA stability than the energy stability of the pump pulse. The reason for this is 
that in the 3-ns window defined by the stretcher bandpass, pump peak power is more representative of the available pump pulse energy and intensity than the integrated energy over the entire pump pulse when the pulse width or the pulse shape of the pump exhibits fluctuations. The resulting peak power stability of the pump was $\pm 2.3 \% \mathrm{rms}$ for 600 shots. As shown previously ${ }^{30}$, operation with slight pump back-conversion yields optimal stability, which can exceed the stability of the pump laser.

\subsection{Peak fluence stability}

For ultrashort pulses $(<10 \mathrm{ps})$, previous studies of damage threshold in dielectrics indicate that the damage threshold is highly deterministic. ${ }^{16}$ It is reasonable to expect that on a small and defect-free area of the dielectric, damage initiation occurs at a point of maximum fluence. It is thus important to study the peak fluence statistics on a shot-toshot basis. Since our damage studies are performed at the beam focus, focal spot size and spectral structure fluctuations must be incorporated into the total measurement error. The primary contributions to the focal spot variability are pump laser spatial and temporal fluctuations leading to variation in near-field OPA beam shape, mechanical movement and vibrations, temperature fluctuations, and air currents. In order to quantify the effect of those perturbations, we have recorded a series of 16-bit images of the focal spot and normalized them to corresponding shot energies. The results are shown in Fig. 8. It is apparent that the hot-spot fluctuation on target is the more significant contributor to fluence variation than the shot-to-shot energy variation. For 12 shots we have determined the rms fluctuation of peak fluence (hot spot) to be $\pm 6.1 \%$. 


\subsection{Parametric fluorescence}

Optical parametric generation or parametric fluorescence (PF) is the equivalent of amplified spontaneous emission (ASE) in lasers, corresponding to parametric amplification of one photon per mode in either the signal or the idler beam. ${ }^{31} \mathrm{PF}$ is emitted in the direction of pump pulse propagation, in a characteristic cone with a spectral composition corresponding to angular phase matching. The range of emitted frequencies is limited by the pump-signal-idler spatial overlap in the crystal for frequencies which are phase-matched at large noncollinear angles. We have characterized the fluorescence of our system by comparing the OPCPA output energy at the damage test setup when the seed pulse to OPCPA is present with the output energy when the seed pulse to OPCPA is blocked. It has been measured that the PF level is reduced by a factor of 3 when the seed pulse is present from the pulse stretcher as a result of the pump pulse depletion in the system. With the seed pulse absent, PF energy measured is $~ 3 \%$ of the total output energy. However, the broad-bandwidth fluorescence is emitted with random phase and is thus stretched by $3 \mathrm{~ns}$ in the pulse compressor. With a fluorescence pulse width of $5.7 \mathrm{~ns}$ corresponding to the pump pulse width, and a 3-ns stretch of the pulse compressor, it is estimated the ratio of the intensity of the recompressed pulse to the PF pedestal intensity level is $\sim 4.4 \times 10^{6}$ for 200 -fs recompressed pulses. This corresponds to

fluences $\sim 1 \mu \mathrm{J} / \mathrm{cm}^{2}$ for our typical damage tests, which is far below the damage threshold for parts under study. Higher PF pedestal contrast could be achieved by utilizing spatial filtering between OPCPA stages. 


\subsection{Beam profiles and beam quality}

The amplified signal is angularly separated from the idler. A 5\% beamsplitter is used to sample the amplified signal beam for spatial and spectral diagnostics. The output face of the second power amplifier crystal is imaged onto a CCD camera, while a spectrometer is used to record amplified signal spectra. The obtained near field amplified signal profile is shown in Fig. 9(a) and closely resembles the pump beam profile. We performed power-in-the-bucket measurement of the encircled pulse energy prior to compression. It was determined that $82 \%$ of the pulse energy is enclosed in a $250-\mu \mathrm{m}$ diameter circle for $\sim \mathrm{f} / 100$ focusing, which can be compared to $\sim 85 \%$ for an ideal top-hat beam of the same diameter. This indicates that the transverse phase aberration on the amplified pulse is relatively small.

We have imaged the focused compressed pulse beam profile on the sample using a 16-bit CCD camera. Typical result is shown in Fig. 9(b). The size of the focal spot on the sample is $250 \mu \mathrm{m}$ at FWHM with $\sim \mathrm{f} / 80$ focusing. The beam focused on the sample exhibits some phase errors, which may be due to wavefront quality of the compressor and beam transport optics. However, this does not influence our damage measurements, since they are based on measurements of the hot spot fluence.

\subsection{Spectral shape variations}

Recompressed pulse shape close to the transform limit is given by the Fourier transform of the amplified pulse spectrum. The majority of our damage studies are performed far from the transform limit by introducing group delay dispersion using our pulse compressor, and in that case the recompressed pulse shape carries a close 
resemblance to the amplified pulse spectrum. Short-pulse laser damage can be influenced by the pulse shape, which has been studied previously. ${ }^{16}$ We have performed single-shot measurements of the amplified pulse spectrum to determine the variation of "hot spots" in the spectrum, which then closely resembles the variation in peak power over the pulse width. The results shown in Fig. 10 for 50 shots show a $\pm 4.9 \%$ rms variation when normalized to the pulse energy. While this effect has some influence on the recompressed pulse shape, the actual pulse shape is determined by the Fourier transform of the spectrum with all additional phase included.

\subsection{Pulse width and shape measurements}

A typical autocorrelation trace is shown in Fig. 11. By comparing the width of the measured autocorrelation trace with the calculated autocorrelation trace based on the transform limit of the amplified pulse spectrum, we estimate the recompressed pulse to be 1.35 times broader than the transform limit, corresponding to a full-width of $205 \mathrm{fs}$. The calculated energy contained in the central peak of the transform-limited pulse based on the measured pulse spectrum is $85 \%$. Probable sources of departure of the recompressed pulse from the transform limit are spectral and spherical aberrations in the pulse stretcher, which contains a plano-convex lens. ${ }^{32}$

For longer pulse durations, we present a typical cross-correlation trace in Fig. 12(a). In the presented example, a 10-ps pulse is cross-correlated with a 200-ps pulse. Our cross-correlation measurement reveals a fine structure of the 10-ps pulse, with approximately 500 -fs wide spikes separated by $30-50 \%$ deep modulations. We trace the origin of this structure to the sharp cutoff of the pulse spectrum in the pulse stretcher. If 
we apply the calculated group-delay dispersion and third-order dispersion spectral phase of the pulse compressor required to produce 10-ps pulses to a top-hat spectrum, the resulting Fourier-transformed pulse shape (Fig. 12(b)) resembles the measured crosscorrelation trace in its overall structure. While some discrepancy remains, this can be explained by the departure of the amplified spectrum from the ideal top-hat shape.

\section{DAMAGE TESTING}

\subsection{Damage observation and results}

We have performed initial damage testing utilizing the short-pulse damage test station. Our measured damage threshold of superpolished, uncoated, fused silica for pulse width of $200 \mathrm{fs}$ and $10 \mathrm{ps}$ are $2.6 \mathrm{~J} / \mathrm{cm}^{2}$ and $5.3 \mathrm{~J} / \mathrm{cm}^{2}$, in close agreement with prior results ${ }^{16}$ that used Ti:sapphire regenerative amplifiers for this measurement.

Occurrence of damage is clearly indicated by an irrerversible, progressive drop of diffraction efficiency on a shot-to-shot basis, corresponding to damage initiation. We have experimentally determined the sensitivity of this method compared to direct observation of damage by a 1000x magnification using a Nomarsky microsope (Fig. 13). The pulse energy level at which damage is observed by monitoring diffraction efficiency is $<10 \%$ higher than the lowest level at which any surface modification is observed using a Nomarsky microscope. (picture) This surface modification can also correspond to conditioning or slight structure modification which gives no measurable effect on diffraction efficiency of the diffraction grating. 


\subsection{Estimate of pulse shape effects on damage threshold}

The dependence of short-pulse laser damage on the shape of the applied pulse is of great importance for validating of results of short-pulse damage testing. The formalism for short-pulse laser damage has been previously developed. ${ }^{16}$ In this model, the shortpulse laser damage is caused by the field-driven multiphoton ionization providing the seed electrons, followed by collisional ionization producing an electron avalanche. An equation can be written which describes the evolution of free electron density in terms of the two contributions: $8^{\text {th }}$ order ionization term corresponding to the minimum energy required to overcome the bandgap in $\mathrm{SiO}_{2}$ for photon energies corresponding to the wavelength of $1053 \mathrm{~nm}$, and the collisional ionization term:

$$
\frac{d n(t)}{d t}=\beta(I(t)) n(t)+P(I(t)) .
$$

The ionization term is dependent on the cross section for $8^{\text {th }}$ order ionization:

$$
P(I)=9.52 \times 10^{10} I^{8} \mathrm{~cm}^{-3} \mathrm{ps}^{-1} .
$$

Collisional ionization is dependent on intensity through the intensity-dependent avalanche rate $\beta(I)$. The $\beta(I)$-dependence for fused silica in the region of intensities of $\sim 1$ $\mathrm{TW} / \mathrm{cm}^{2}$ has been fitted to

$$
\beta(I)=10\left(\frac{I}{1 \mathrm{TW} / \mathrm{cm}^{2}}\right) \mathrm{ps}^{-1}
$$

The calculation of the free electron density then proceeds through simple numerical solution of the differential equation (1). It is important to define a characteristic free electron density which corresponds to damage. Here we use the critical plasma density,

$$
n_{c}=m \varepsilon_{0} \frac{\omega^{2}}{e^{2}},
$$


as a characteristic damage threshold electron density. While this choice may not correspond to the actual macroscopic damage, it is still useful in estimating the relative impact of the pulse shape on short-pulse laser damage. The calculated critical plasma density is $n_{c}=1 \times 10^{21} \mathrm{~cm}^{-3}$.

This model can be used to estimate the effect of fast modulation of the 10-ps recompressed pulse such as the one measured on PDTS. We first use a top-hat pulse shape, with an assumption that the intensity is a constant during the pulse duration. In the calculation, a 20-th order supergaussian is used. For the fluence of $5 \mathrm{~J} / \mathrm{cm}^{2}$ we obtain a peak intensity of $0.5 \mathrm{TW} / \mathrm{cm}^{2}$. The resulting free electron density is shown in Fig. 14(a). The point $\mathrm{n}_{\mathrm{e}} \sim \mathrm{n}_{\mathrm{c}}$ is reached at $\mathrm{t}=1.5 \mathrm{ps}$, while the final $\mathrm{n}_{\mathrm{e}}=1.6 \times 10^{28} \mathrm{~cm}^{-3}$. The calculated fluence that gives the final $\mathrm{n}_{\mathrm{e}} \sim \mathrm{n}_{\mathrm{c}}$ is $3.5 \mathrm{~J} / \mathrm{cm}^{2}$.

We can apply calculated spectral phase up to the cubic order to obtain a better representation of the actual pulse shape when the compressor is displaced from the ideal separation for transform-limited pulse generation:

$$
\phi^{\prime \prime}=-2.1 \times 10^{5} \mathrm{fs}^{2} / \mathrm{rad}, \phi^{\prime \prime \prime}=+1.7 \times 10^{6} \mathrm{fs}^{3} / \mathrm{rad}^{2} .
$$

The result is shown in Fig. 14(b). The resulting non-transform-limited pulse shape has an envelope which is a close representation of the pulse spectrum. However, the strong ( $40 \%)$ modulation is present inside the pulse envelope, primarily as the result of the hard spectral clip. This modulation is similar to the one measured using a cross-correlator on PDTS. For the fluence of $5 \mathrm{~J} / \mathrm{cm}^{2}$ we obtain a peak intensity of $0.76 \mathrm{TW} / \mathrm{cm}^{2}$. The resulting free electron density is shown in Fig. 13(b). The point $n_{e} \sim n_{c}$ is reached at $t=2.1$ ps, while the final $n_{e}=2 \times 10^{27} \mathrm{~cm}^{-3}$. The calculated fluence that gives the final $\mathrm{n}_{\mathrm{e}} \sim \mathrm{n}_{\mathrm{c}}$ is 3.7 $\mathrm{J} / \mathrm{cm}^{2}$ 
The fine structure and modulation of the temporal profile seems to have a slight effect on the total number of generated electrons for fluences beyond the damage threshold. However, even though the peak power increase can be near $40 \%$ compared to smooth top-hat pulses, the fluence required for $\mathrm{n}_{\mathrm{e}} \sim \mathrm{n}_{\mathrm{c}}$ is nearly constant. This indicates that this type of modulation, characteristic for a clipped spectral profile with additional large group delay, has little effect on the damage threshold.

\section{CONCLUSION}

In conclusion, we have developed and characterized a highly stable, nearly degenerate OPCPA system pumped by a commercial pump laser. We have characterized OPCPA performance characteristics relevant to its use as a precision diagnostic tool for short-pulse damage testing. We have incorporated OPCPA into a short-pulse damage test setup, allowing measurements of optic damage thresholds and optic surface modification by ultrashort pulses with variable pulse width, ranging from $200 \mathrm{fs}$ to $>10 \mathrm{ps}$. Information has been obtained on short-pulse optical damage of dielectric- and metal-coated optics, of importance for future HEPW lasers and all-OPCPA lasers.

The overall accuracy of our measurement for multi-shot experiments is determined by aggregate fluctuations in space and in time. We have characterized all relevant stability parameters of our system for operation both near transform limit of the amplified pulse spectrum, and for longer ( 10-ps) pulse operation. An important limitation with regards to the precision short-pulse damage station operation in air has been identified. Nonlinear phase accumulation (B-integral) limits this operation to f-numbers no greater 
than $\sim \mathrm{f} / 100$. For higher peak power studies, it is necessary to perform the damage tests in vacuum to avoid self-focusing effects.

A trade-off of our design that we have identified is the system stability/efficiency and reduction of modulation and ringing on the recompressed pulse due to spectral broadening and hard spectral clip in the stretcher or compressor. When used as a front end of a Nd:glass laser, this trade-off is typically not encountered due to spectral narrowing of Nd:glass amplifiers, leading to smooth, bell-shaped amplified spectra. However, future all-OPCPA systems utilizing top-hat or supergaussian pump pulses will have their peak power and contrast reduced if the system is running in a stable, highpump depletion mode. To avoid the reduction of contrast, high-conversion- efficiency, all-OPCPA systems for high intensity experiments will have to be pumped by bell-shaped pump pulses, which will mandate active or passive spatiotemporal shaping between the amplifier stages to arrive with the bell-shaped amplified signal and high extraction of the pump pulse.

Our short-pulse damage setup has been used to measure the effects of multi-pulse surface modification and damage threshold. In the future, single-shot damage studies will be implemented by acquiring the pulse exact spatial characteristics on each shot.

\section{ACKNOWLEDGEMENTS}

This work was performed under the auspices of the U. S. Department of Energy by the University of California, Lawrence Livermore National Laboratory under Contract No. W-7405-Eng-48. 


\section{LIST OF REFERENCES}

The importance and motivation for development of high-energy petawatt lasers has been recently outlined in a report to the U.S. Congress by the National Nuclear Security Administration, entitled "High Enegy Petawatt Lasers and the Stockpile Stewardship Program".

J. D. Kmetec, C. L. Gordon, J. J. Macklin, B. E. Lemoff, G. S. Brown, and S. E. Harris, Phys. Rev. Lett. 68, 1527 (1992).

S. C. Wilks and W. L. Kruer, IEEE J. Quantum Electron. 33, 1954 (1997).

M. Tabak, J. Hammer, M. E. Glinsky, W. L. Kruer, S. C. Wilks, J. Woodworth, E.

M. Campbell, M. D. Perry, and R. J. Mason, Phys. Plasmas 1, 1626 (1994).

Development of high-efficiency, high damage threshold diffraction gratings for HEPW pulse compressors is under way at the Lawrence Livermore National Laboratory (Livermore, California, USA), General Atomics (San Diego, California, USA), and Jobin-Yvon (France). N. Bloembergen, IEEE J. Quantum Electron. 10, 375 (1974). 23, 2144 (1980).

12 D. Du, X. Liu, G. Korn, J. Squier, and G. Mourou, Appl. Phys. Lett. 64, 3071 (1994). 
B. C. Stuart, M. D. Feit, A. M. Rubenchik, B. W. Shore, and M. D. Perry, Phys. Rev. Lett. 74, 2248 (1995). D. v. d. Linde and K. Sokolowski-Tinten, Appl. Surf. Sci. 154, 1 (2000).

A. C. Tien, S. Backus, H. Kapteyn, M. Murnane, and G. Mourou, Phys. Rev. Lett. 82, 3883 (1999). Perry, Phys. Rev. B 53, 1749 (1996).

A. Dubietis, G. Jonusauskas, and A. Piskarskas, Opt. Commun. 88, 437 (1992).

I. N. Ross, P. Matousek, M. Towrie, A. J. Langley, and J. L. Collier, Opt. Commun. 144, 125 (1997).

19 X. D. Yang, Z. Z. Xu, Y. X. Leng, H. H. Lu, L. H. Lin, Z. Q. Zhang, R. X. Li, W. Q. Zhang, D. J. Yin, and B. Tang, Opt. Lett. 27, 1135 (2002). J. Collier, C. Hernandez-Gomez, I. N. Ross, P. Matousek, C. N. Danson, and J. Walczak, Appl. Opt. 38, 7486 (1999).

21 I. Jovanovic, B. J. Comaskey, C. A. Ebbers, R. A. Bonner, D. M. Pennington, and E. C. Morse, Appl. Opt. 41, 2923 (2002). H. Yoshida, E. Ishii, R. Kodama, H. Fujita, Y. Kitagawa, Y. Izawa, and T. Yamanaka, Opt. Lett. 28, 257 (2003). L. J. Waxer, V. Bagnoud, I. A. Begishev, M. J. Guardalben, J. Puth, and J. D. Zuegel, Optics Letters 28, 1245 (2003). M. J. Guardalben, J. Keegan, L. J. Waxer, V. Bagnoud, I. A. Begishev, J. Puth, and J. D. Zuegel, Opt. Exp. 11, 2511 (2003). 
F. Rotermund, V. Petrov, F. Noack, V. Pasiskevicius, J. Hellstrom, E. Laurell, H. Hundertmark, P. Adel, and C. Fallnich, Electron. Lett. 38, 561 (2002). A. Galvanauskas, A. Hariharan, D. Harter, M. A. Arbore, and M. M. Fejer, Opt. Lett. 23, 210 (1998). I. Jovanovic, J. R. Schmidt, and C. A. Ebbers, Appl. Phys. Lett. 83, 4125 (2003). D. M. Pennington, C. G. Brown, T. E. Cowan, S. P. Hatchett, E. Henry, S. Herman, M. Kartz, M. Key, J. Koch, A. J. MacKinnon, M. D. Perry, T. W. Phillips, M. Roth, T. C. Sangster, M. Singh, R. A. Snavely, M. Stoyer, B. C. Stuart, and S. C. Wilks, IEEE J. Sel. Top. Quantum Electron. 6, 676 (2000). D. J. Armstrong, W. J. Alford, T. D. Raymond, A. V. Smith, and M. S. Bowers, J. Opt. Soc. Am. B 14, 460 (1997). S. K. Zhang, M. Fujita, M. Yamanaka, M. Nakatsuka, Y. Izawa, and C. Yamanaka, Opt. Commun. 184, 451 (2000). D. A. Kleinman, Phys. Rev. 174, 1027 (1968). A. Sullivan and W. E. White, Opt. Lett. 20, 192 (1995). 


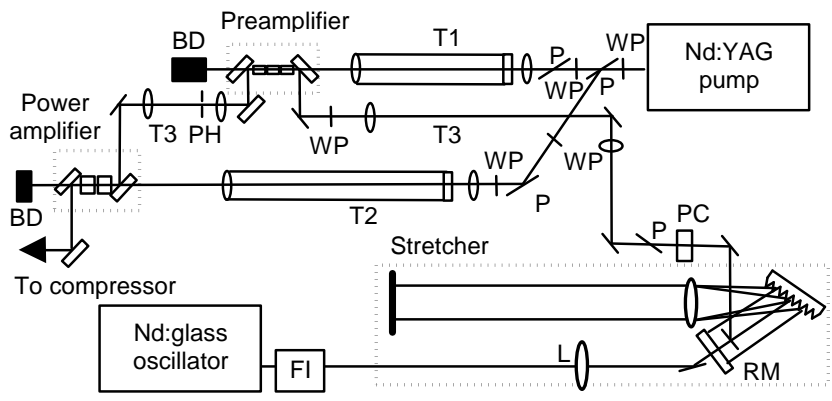

Figure 1

I. Jovanovic, Review of Scientific Instruments 


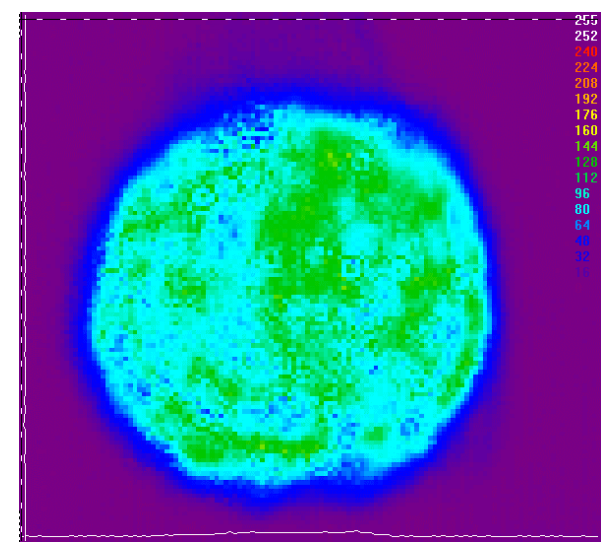

Figure 2

I. Jovanovic, Review of Scientific Instruments 


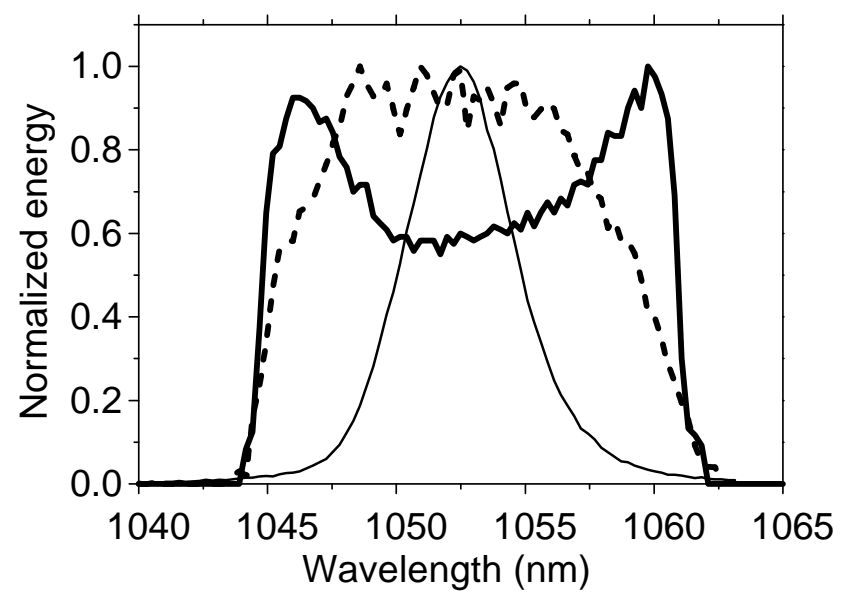

Figure 3

I. Jovanovic, Review of Scientific Instruments 


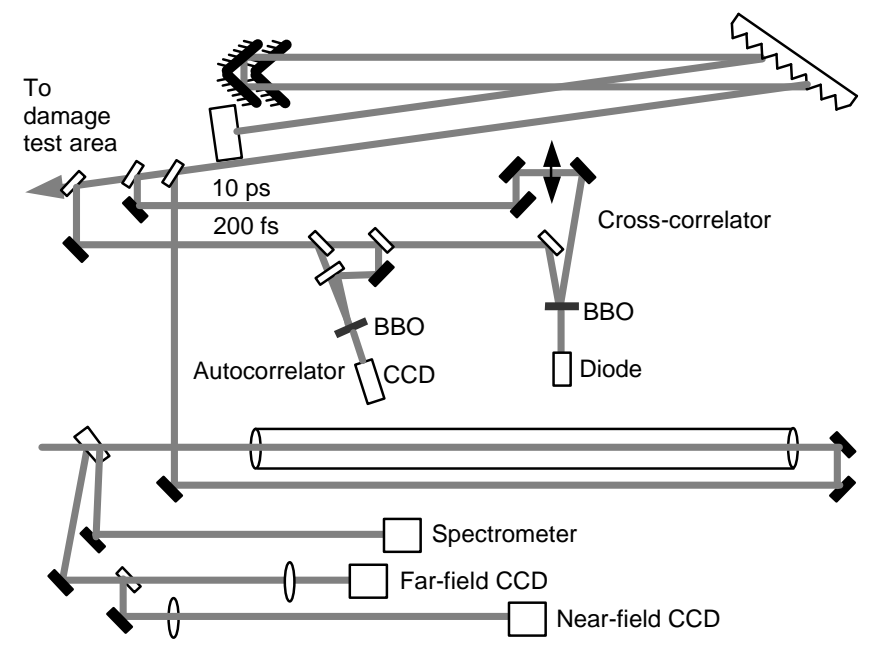

Figure 4

I. Jovanovic, Review of Scientific Instruments 


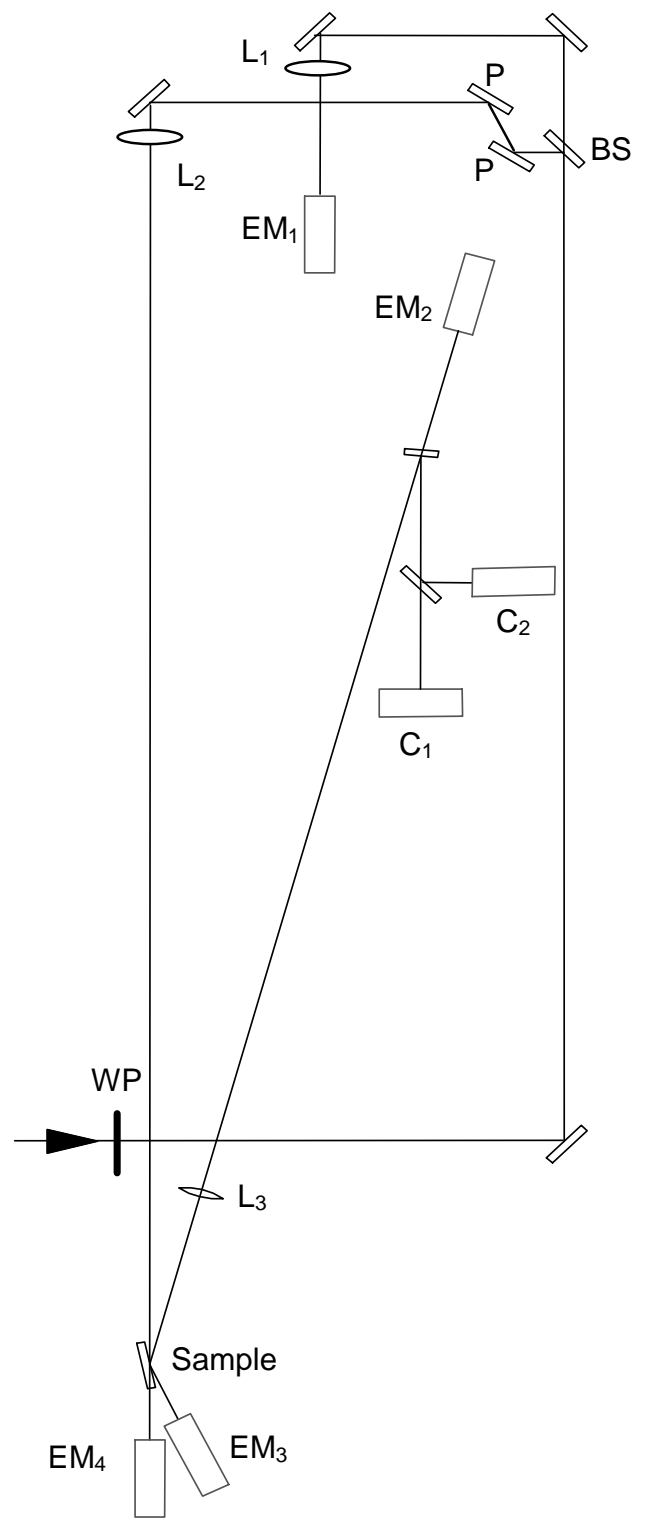

Figure 5

I. Jovanovic, Review of Scientific Instruments 


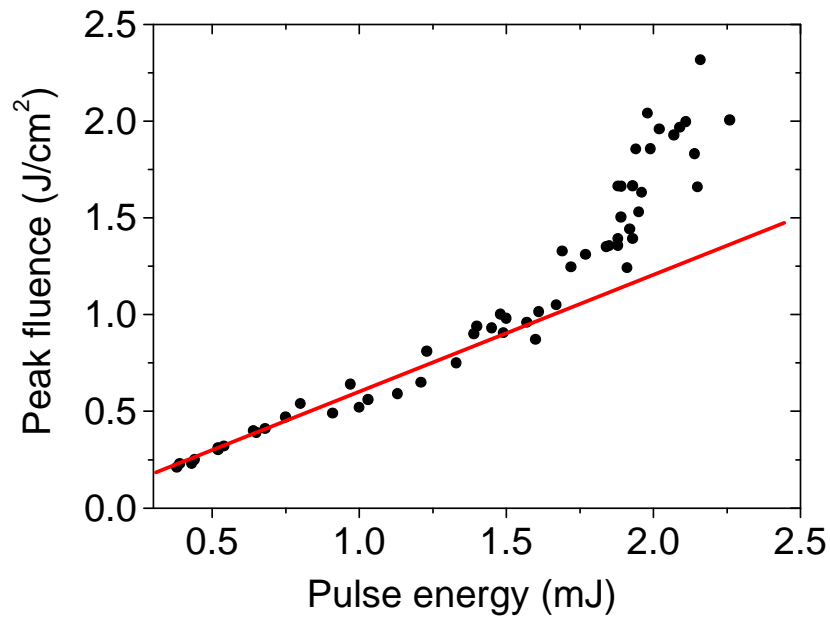

Figure 6

I. Jovanovic, Review of Scientific Instruments 


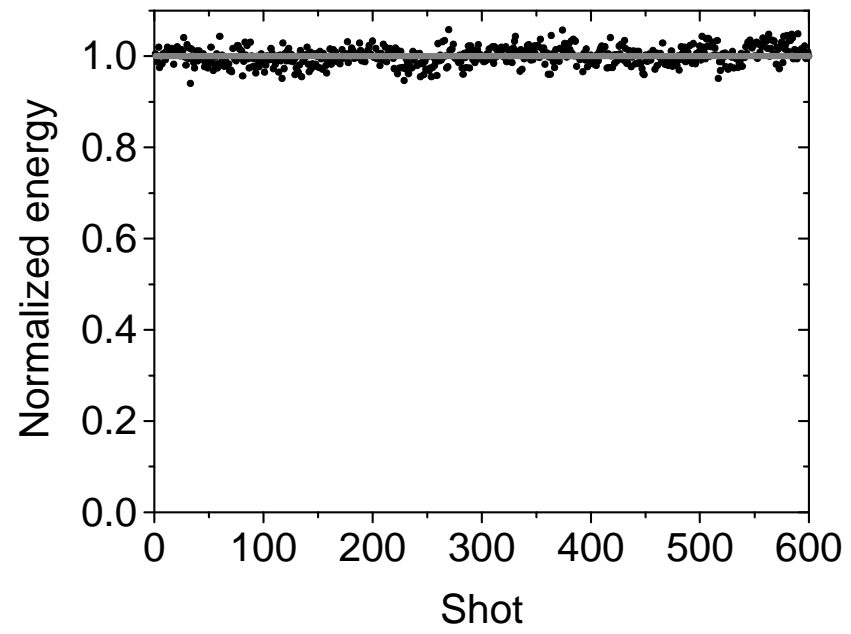

Figure 7

I. Jovanovic, Review of Scientific Instruments 


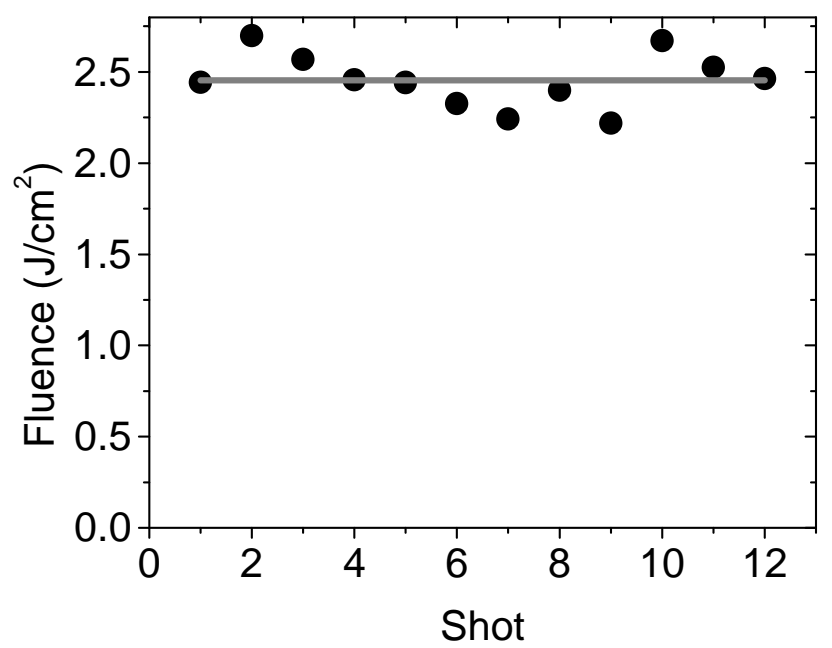

Figure 8

I. Jovanovic, Review of Scientific Instruments 


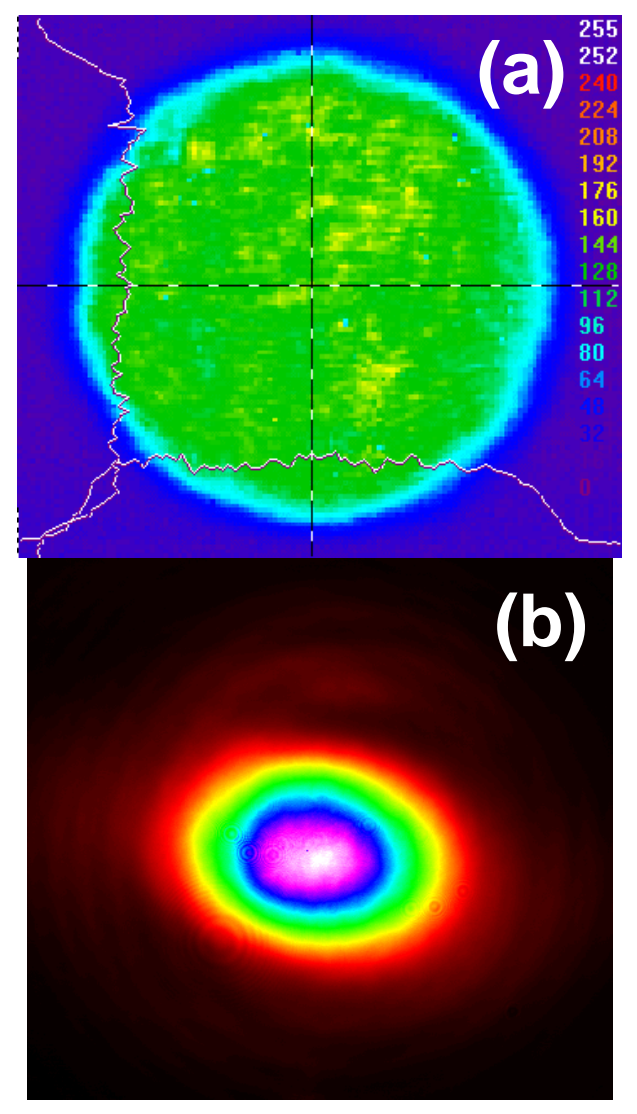

Figure 9

I. Jovanovic, Review of Scientific Instruments 


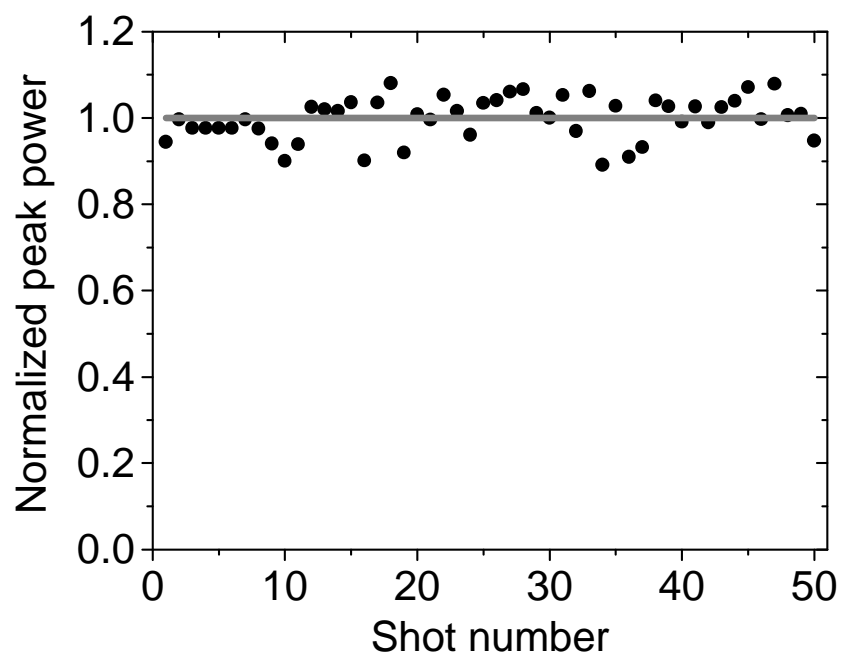

Figure 10

I. Jovanovic, Review of Scientific Instruments 


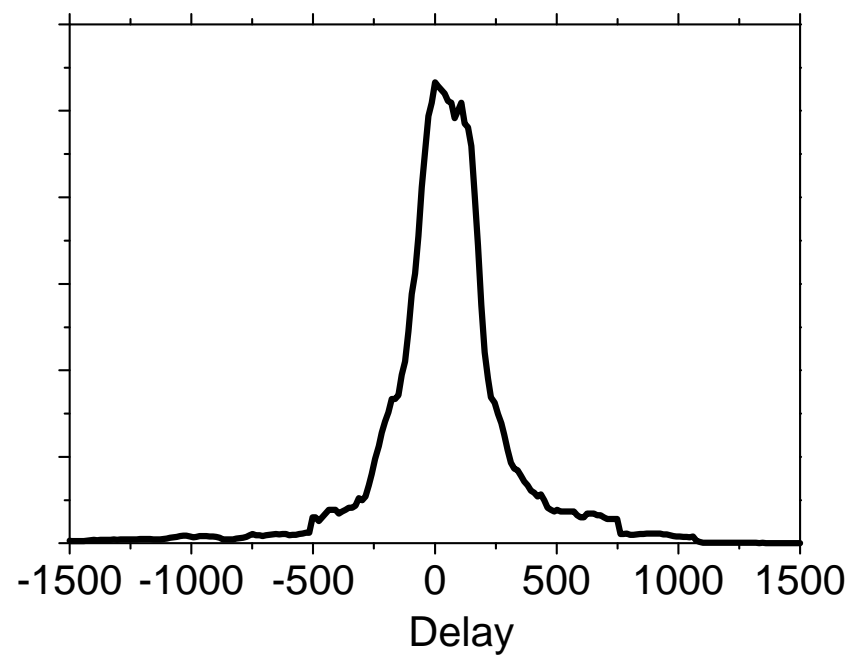

Figure 11

I. Jovanovic, Review of Scientific Instruments 

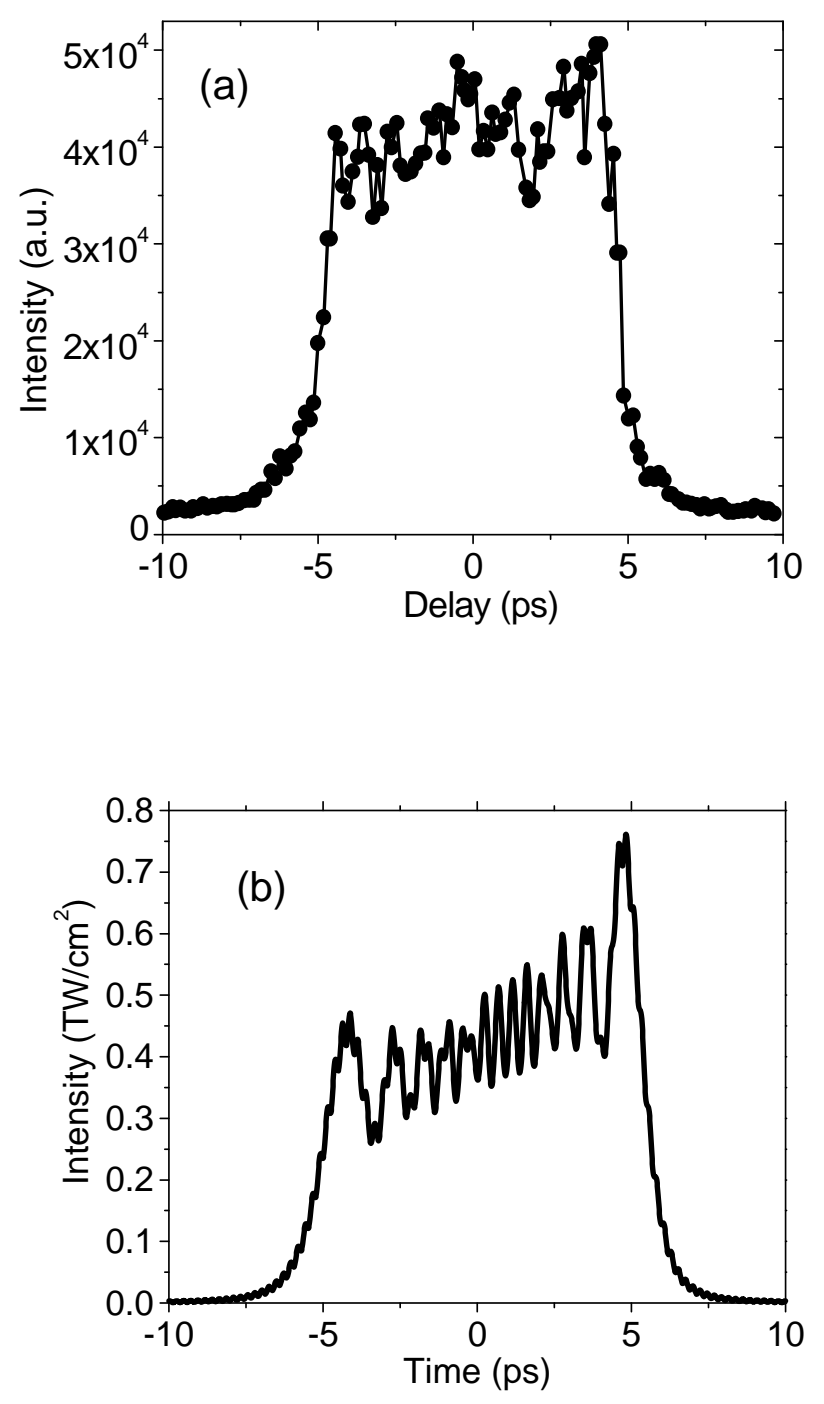

Figure 12

I. Jovanovic, Review of Scientific Instruments 


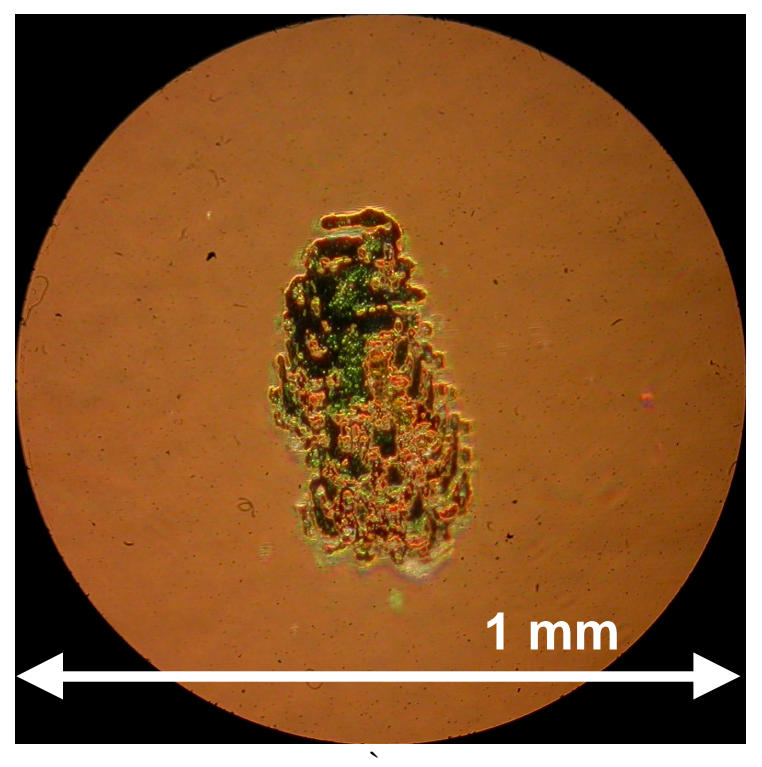

Figure 13

I. Jovanovic, Review of Scientific Instruments 

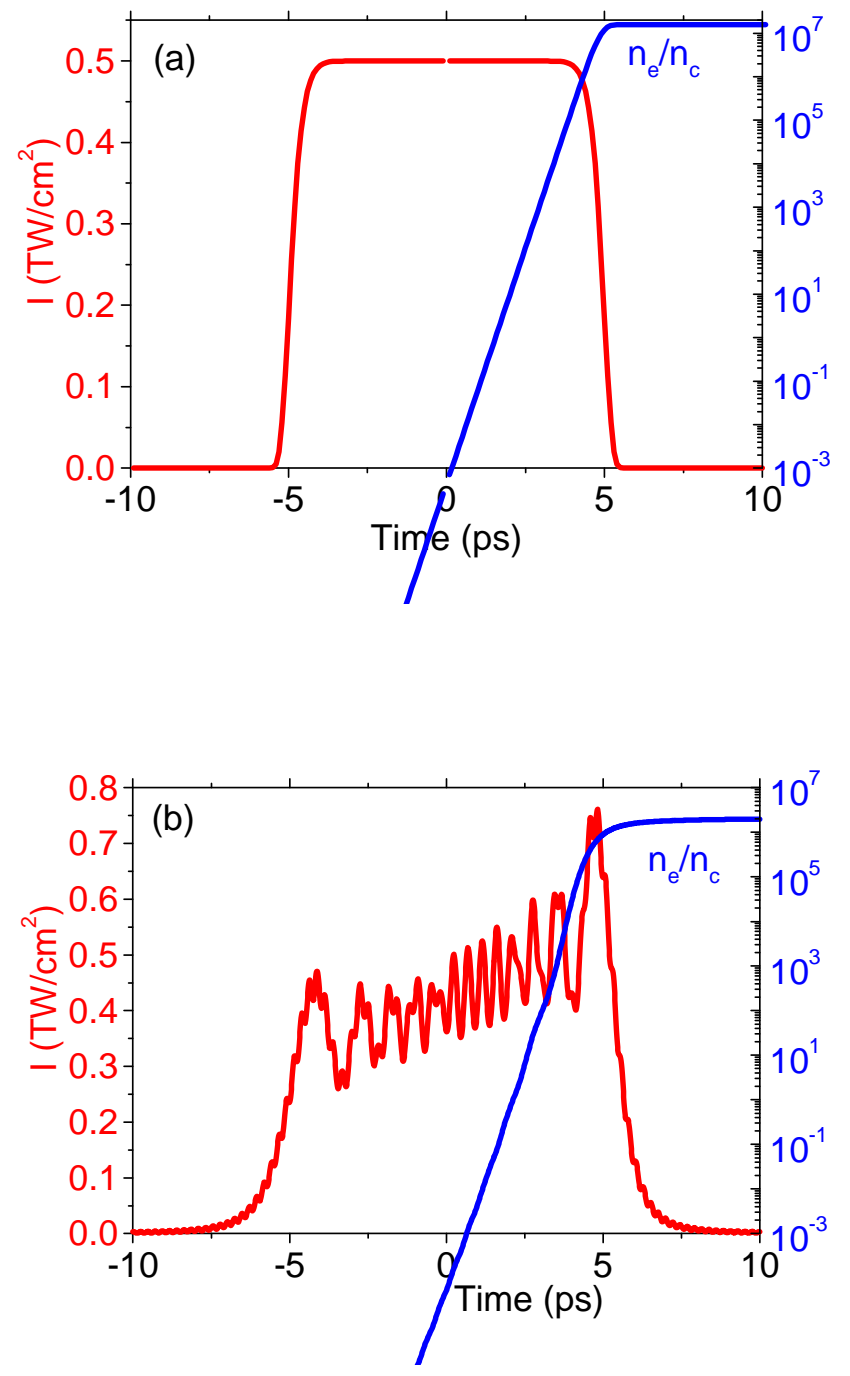

Figure 14

I. Jovanovic, Review of Scientific Instruments 


\section{LIST OF FIGURE CAPTIONS}

Fig. 1. OPCPA system used for short-pulse damage testing, excluding the pulse compressor. P-polarizer, L-lens, WP- $\lambda / 2$ waveplate, BD-beam dump, T-telescope, PHpinhole, FI-Faraday isolator, RM-roof mirror, PC-Pockels cell.

Fig. 2. Transverse spatial profile of the pump pulse at OPA crystals is supergaussian

Fig. 3. Input (thin solid line) and amplified spectra obtained from the power amplifier at 40-mJ (dashed line) and 55-mJ (thick solid line) level. Pump depletion occurs first in the center of the spectrum, leading to spectral broadening.

Fig. 4. Pulse expanding telescope and double-compressor setup with intensity autocorrelator and cross-correlator.

Fig. 5. Short-pulse damage setup. EM-energy meter, BS-beamsplitter, L-lens, P-polarizer, WP- $\lambda / 2$ waveplate, $\mathrm{C}_{1}$ - 16 -bit CCD camera, $\mathrm{C}_{2}-8$-bit CCD camera.

Fig. 6. Measurement of peak fluence as a function of pulse energy with the short-pulse damage setup (Fig. 5) that utilizes lens L with focal length $\mathrm{f}=4 \mathrm{~m}$ with 200 -fs pulses. Shown with a solid line is the fit to data for energies $<1.5 \mathrm{~mJ}$. Self-focusing effects become apparent when the energy is increased above $\sim 1.5 \mathrm{~mJ}$. Our setup overcomes this problem by utilizing a shorter focal length lens $\mathrm{L}(\mathrm{f}=2 \mathrm{~m})$.

Fig. 7. Measurement of OPCPA stability for 600 shots (1 minute of operation). Energy is normalized to mean energy. Standard deviation is $1.9 \%$.

Fig. 8. Variation of the hot spot fluence in the far field as recorded by a 16-bit CCD

camera. The minimum spatial resolution element in the measurement is $5.8 \mu \mathrm{m}^{2}$. The calculated variation is $\pm 6.1 \% \mathrm{rms}$. 
Fig. 9. Transverse spatial profile of the amplified signal beam at the output of the power amplifier in (a) near field, and (b) far field (FWHM beam diameter 250 $\mu \mathrm{m}$ ).

Fig. 10. Variation of peak amplified spectral intensity for 50 shots. Calculated standard deviation is $4.9 \%$.

Fig. 11. Autocorrelation trace of the short diagnostic pulse. Minimum pulse width is 205 fs.

Fig. 12. (a) Measured cross-correlation trace of a 10-ps recompressed pulse; (b) calculated cross-correlation trace by applying the compressor quadratic and cubic dispersion to a top-hat spectrum.

Fig. 13. Damage site on a diffraction grating magnified using a Nomarsky microscope. Fig. 14. Calculated free electron density for (a) 20-th order supergaussian, 10-ps pulse, and (b) pulse produced by applying quadratic and cubic phase terms from the pulse compressor on a 20-th order supergaussian, 16-nm FWHM hard-clipped spectrum. Fluence is $5 \mathrm{~J} / \mathrm{cm}^{2}$. 